\title{
Morphometry Applied to the Study of Morphological Plasticity During Vertebrate Development
}

\author{
Christina Wahl \\ Wells College, Aurora, NY \\ USA
}

\section{Introduction}

Embryos and young growing animals do not develop in isolation. The lake shiner, Notropis atherinoides, and the blue-gill sunfish, Lepomis incisor, produce more body segments when raised in cool water. Chickens raised in constant light have flattened corneas, making them abnormally hyperopic, or "far-sighted". Cichlids provided with different types of diets during early growth develop different jaw morphologies as adults. Craniofacial proportions are different in children born with fetal alcohol syndrome, and those born to women who smoke tend to be underweight compared to the average. These examples demonstrate the plasticity of shape and size that is possible during ontogeny as a result of environmental conditions, and all produce permanent effects on the adult phenotype. In this chapter, I will describe different forms of vertebrate developmental phenotypes and phenotypic plasticity, with a brief review of the relevant biology, and then I will present some preliminary approaches to morphometric quantification of these phenomenae.

The metamorphosis of a unique embryonic, or larval, body type into the definitive adult body form of the species (such as seen in fishes) involves dramatic, permanent phenotypic change, whereas regeneration, a property that the embryos of many species possess to a remarkable degree, is a form of phenotypic plasticity that effects embryonic repairs. Metamorphosis is highly refined among the invertebrates, in particular among insects, however some vertebrates (fishes) exhibit metamorphosis too, and quite spectacularly (see Figure 1). Among the mesopelagic Stomiiform fishes, larval craniofacial features include elaborate larval eyestalks and elongated, dorso-ventrally flattened skulls, which transform during metamorphosis into a more typical face...eyes seated within orbital sockets instead of at the ends of eye stalks... and increased skull depth. Some species of fishes actually shrink in size as well as change their shape during metamorphosis. For instance, the leptocephalus larvae of anadromous eels is significantly larger than the adult of the species.

Embryonic regeneration is spectacular...it is the ability to achieve scarless reconstruction of injured body parts... and can extend from replacement of missing limbs to functional repair of enucleated eyes, as noted among amphibians in the order Urodela. The ability to 
regenerate is more robust among embryos and among the young, although some vertebrates, such as the urodelans, retain vigorous regenerative capacity throughout life.

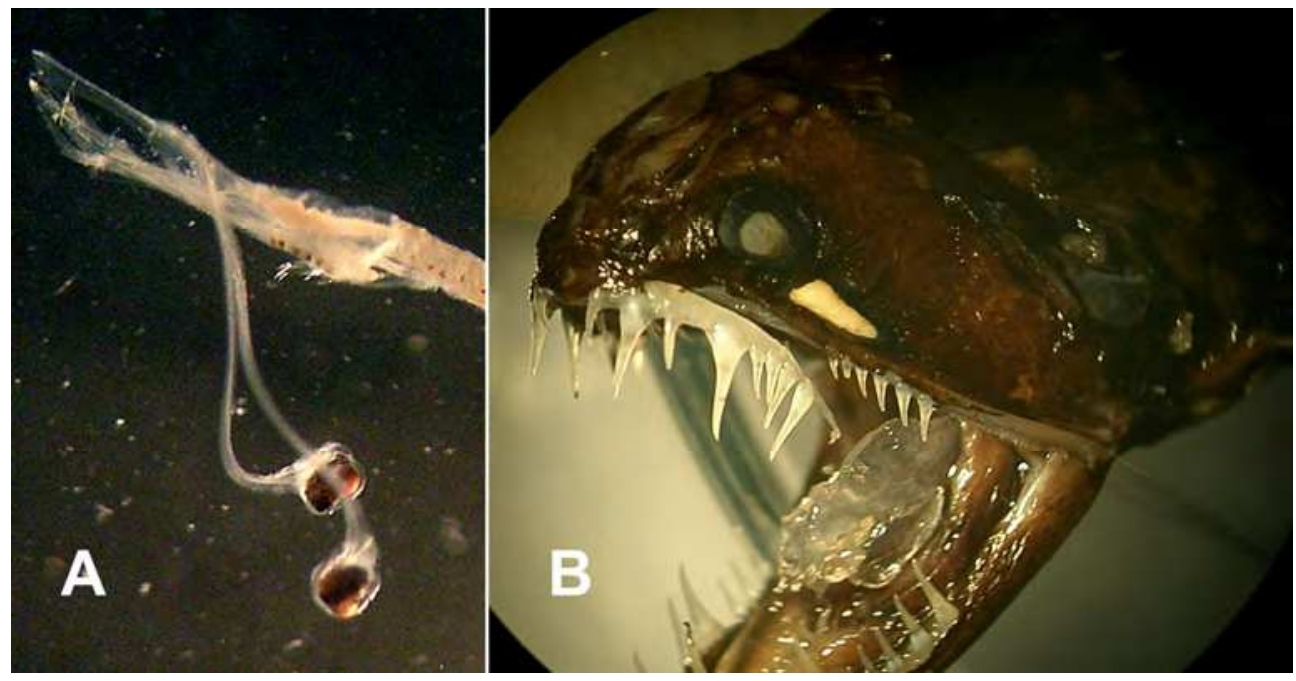

Fig. 1. Larval dragonfish Idiacanthus atlanticus (a) standard length (SL) $3.5 \mathrm{~cm}$, and adult (b) SL $13 \mathrm{~cm}$. Craniofacial metamorphosis is pronounced, especially around the periocular area. Larval eye stalks are up to $1 / 3$ of total body length, but are absent in the adult. The adult specimen shown here has partially ingested a jelly, still visible in its throat (Specimens photographed by C. Wahl at CSIRO, Hobart, and at the Australian Museum, Sydney, Australia).

Although gene expression is at the heart of development and determines the basic "bauplan", specific details of morphology such as size, shape, numbers of body segments, and even sex can be strongly influenced by the embryonic and early life environment, principally through action on signaling pathways or through gene regulation. In recent years, new attention has been paid to the ways in which developmental mechanisms are able to produce specific phenotypic solutions to environmental variables (Müller, 2011).

Flexibility to adapt the body to local conditions during growth confers on the embryo, larva, or juvenile an opportunity to fine-tune certain aspects of anatomy and physiology and may increase fitness as the individual reaches adulthood. Epigenetic influence on development may prove to be not only common, but in many cases, critical to adaptive evolutionary change.

Whereas the healing of amputated limbs or enucleated eyes in salamanders can result in slightly smaller yet functional replacements (Wahl, 1985), among embryos perfectly scaled repairs are possible (Wahl and Noden, 2001). The mechanisms active during embryonic regeneration must co-exist in the same body with temporally disparate ontogenetic activity.... because one part of the body is being completely re-built while the rest is already further along the road towards adulthood.

Development is defined, for the purposes of this chapter, as the process of transformation of haplo-diploid organisms from zygote to sexual maturity. The "embryonic period" is defined 
as the time during development when the organism is unable to live without either a yolk sac or a placenta. The term "fetal" is used most often by medical professionals in reference to the latter period of human gestation, but since the exact developmental interval it refers to is poorly defined and does not apply to other vertebrates, the term is not useful to the basic science of developmental biology.

Morphometry, i.e., methods used to quantify body forms, can be usefully applied to many questions in development, ecology, and physiology. Morphometric assessment of developing organisms offers valuable insights into the consequences of both natural and pathological environmental variables, and usefully informs analysis of epigenetic influence on gene expression patterns. This chapter introduces the different types of morphological variation among vertebrate embryos, and discusses some morphometric assessment techniques. As a colleague has pointed out, "It may be worth noting in passing that shape is a qualitatively discrete character; it is only our insufficient description of it that forces us to rely on continuous measures." (McCune, 1981) . Quantitative measurements allow us to evaluate incremental changes in shape and size, both without and within bodies and organs.

\section{Adaptive and maladaptive morphological plasticity}

Growth is either uniform or allometric (disproportionate), and each type occurs naturally both within and among species. An example of uniform growth is bilateral symmetry. However, the developmental bauplan also necessitates allometry; limbs cannot grow properly without the prior appearance of the nervous and circulatory systems. The vertebrate head is usually disproportionately large throughout the embryonic period in order to prioritize development of the brain, eyes, and mouthparts. Allometry as a manifestation of morphological integration occurring during development has been studied for many years (Klingenberg, 2008).

Environmental stressors alter growth patterns, and this is important to recognize and quantify morphometrically. It may also be important to distinguish between stressors that affect uniform growth versus those that influence allometric growth, since these have different implications to both short and long term fitness and viability.

\subsection{Uniform growth}

As D'Arcy Thompson pointed out in 1917, with respect to biological systems, as an organism increases in size, the forces in action within its systems vary. For instance, some physical features scale as functions of the mass, others scale with volume. While the "dimensions" may remain the same in our equations of equilibrium, the relative values alter with scale (Bonner, 1969). The consequences of this "principle of similitude", first described by Galileo, has implications at every level of the developing body. Thus, gravity is of consequence to the whole animal only after it reaches a certain size, putting constraints on the maximum size attainable--but gravity is not a significant force to the neurulating embryo, where other properties such as diffusion gradients and turgor pressure are more important. The young embryo relies on direct diffusion of oxygen to the tissues prior to the development of its circulation, and makes use of turgor pressure to expand the brain and create various body folds. Such forces as viscosity and surface tension can have enormous influence on body form during this period. 
Aside from genetic malfunctions, variations of developmentally significant environmental parameters produce asymmetries of uniform scaling, known as "fluctuating asymmetry" (Van Valen, 1962). Because bilateral asymmetries originate from random perturbations of developmental processes (Klingenberg, 2003), such asymmetries must arise within the developmental pathways themselves. Possibly because symmetry is a measure of developmental stability, among vertebrates it has been shown that bilaterally symmetrical individuals are more attractive than asymmetrical individuals to members of the opposite sex (Etcoff, 2000).

What is the scholarly interest in attending to variations of uniform scaling among developing organisms? In the evolutionary context, it is because interactions between developmental pathways have significant effects on the phenotypic outcome and stabilizing selection should limit variation. However, adaptive plasticity to environmental parameters is also a survival strategy and is the mechanism by which the choice is made to mature at smaller or larger body sizes, a response to limited resources known as the "thrifty phenotype".

"The thrifty phenotype is the consequence of three different adaptive processes - niche construction, maternal effects, and developmental plasticity... The three processes also operate at different paces... In contemporary populations, the sensitivity of an offspring's development to maternal phenotype exposes the offspring to adverse effects, through four distinct pathways. The offspring may be exposed to (1) poor maternal metabolic control (e.g. gestational diabetes), (2) maternally derived toxins (e.g. maternal smoking), or (3) low maternal social status (e.g. small size)." (Wells, 2007).

During nutritional dearth, an individual may complete development at a smaller body size or mass than when the nutritional status is excellent. Smaller, metabolically less active individuals produced on limited nutrition exhibit this "thrifty phenotype", demonstrated in several species, including rats (Buresova et al., 2006). Understanding how the thrifty phenotype is generated, and what the long-term consequences of such a phenotype might be, is currently of great interest due to the rising incidence of obesity and type 2 diabetes among western civilizations (Wells, 2007). These "diseases of the wealthy" are regarded by some as a maladaptive response to calorie-rich but nutritionally inadequate prenatal diets, where offspring, like their mothers, continue to consume more calories than their "thrifty" metabolism is equipped to burn.

\subsection{Allometric growth}

Normal developmental patterns of allometry and variations due to selection pressures are a topic of long-standing interest to evolutionary biologists, spawning the field of "evo-devo". Some growth patterns may be a "normal" consequence of the immediate environment, for instance, differences correlated with temperature include shorter limbs among endotherms at higher latitudes (Allen's Rule) for which a possible mechanism has recently been discovered... mice raised at lower temperatures have shorter limbs than littermates raised at higher temperatures (Serrat et al., 2008). Another example is that more body segments differentiate among fish of a given species developing in cool water, than are found in conspecifics raised in warmer water. Further study of this meristic and others correlated with temperature might reveal whether Bergman's Rule (the reduction of surface-to-volume ratio with reduced environmental temperature) or Allen's Rule (shorter limbs at lower 
environmental temperatures) are manifestations of epigenetic responses by the developing animal. There are also pragmatic reasons to count fish vertebrae or make other measurements of animals from different climates. This information could be of assistance, along with DNA fingerprinting, in identifying fishing violations, or in determining where a particular animal "grew up".

Thompson defined allometry as "the study of size and its consequences" (Bonner, 1969), and there are physiological consequences of size and scale within the developing organism that are related to constraints of integration of developing body systems. Many allometric relationships scale as the power function $y=b x$, where $\mathrm{x}$ and $\mathrm{y}$ are the two traits being compared (for instance, height and weight), b is the y-intercept, and $a$ is the slope. However, any linear relationship with a y-intercept greater than 0 can describe allometric growth. For a detailed discussion of the use, and misuse, of mathematical relationships that describe allometric growth the reader is referred to many excellent reviews on the subject, for instance, (Gould, 1966).

The response of developing organisms to local environment with phenotypic adaptation has been termed "epigenetic innovation".

"The fact that perturbations of general developmental parameters, such as blastema size, timing of processes, inductive interactions, or cell division rates, could yield very specific morphogenetic results that (mimic) patterns observed in natural change (is) a strong indication that the rules of ontogenetic development (have) an impact on the process of evolutionary variation." (Müller, 2011).

This idea has profound implications when one considers how developmental pathologies arise as a result of conditions such as hypoxia, hypertension, hyperglycemia, and the like. If embryos are capable of "epigenetic innovation" in a single generation, then environment.... influenced, among amniotes, by such factors as maternal diet and behavior....will affect both the morphological and physiological phenotype of the offspring, just as external environmental parameters like temperature directly affect the development of poikilotherms.

Examples of abnormal variations in the embryonic environment include placental insufficiencies, fungal/viral infections, and teratogens. The effects of these unusual environmental parameters on embryonic morphometrics may not be direct, but can be mediated through changes in embryonic behavior patterns. For instance, mechanical forces influence formation of bones and cartilage, hence "phenotypic plasticity" of the skeleton (Müller, 2003), so reduced embryonic motility will produce skeletal insufficiencies (Hall and Herring, 1990). The responsiveness of skeletogenesis to embryonic movements means that there is a genetic permissiveness for de novo formation of skeletal elements in the embryo, a phenomenon we have often observed while performing experiments in the study of avian craniofacial morphogenesis (Wahl and Noden, 2001).

The variance of maladaptive phenotypic expressions is often greater than "normal". This confounds to some degree the ability to determine whether the response is primary or secondary to the perturbation. One approach utilizes a strain of animals with a mutation in the somatic growth axis as a second control when making morphometric comparisons (Boughner et al., 2008). 


\section{Morphological responses by embryos and growing vertebrates to environmental variables}

Although many environmental variables are known to influence developmental phenotypes, here I will discuss just two: the effects of oxygen tension on overall craniofacial development, and the effects of ambient light on the shape and ultimate size of the developing eye. The reader will find many other examples in the literature on topics such as: the effects of light on pigmentation and neuromast distribution; the effects of environmental organophosphates on limb development, differentiation of the reproductive organs, and rate of sexual maturation; the influence of gravity on early body patterning; and the effects of temperature on sex determination in reptiles.

\subsection{Responses to hypoxia}

At early embryonic stages, oxygen effects on cell proliferation and differentiation are different from those in the adult. For example, low oxygen tension is critical to certain aspects of normal development and cell differentiation, such as neurulation and chondrogenesis. Each type of embryonic tissue responds uniquely to local variations in oxygen tension (Huang et al., 2004; Webster, 2007). Thus, mesenchymal condensations destined to give rise to endochondral skeletal elements and joints normally show marked hypoxia compared to neighboring tissue during early embryogenesis, and will not differentiate if local $\mathrm{O}_{2}$ concentrations are too high (Provot et al., 2007; Thompson et al., 1989). Angioblasts, highly migratory cells that aggressively cross tissue boundaries during embryogenesis, retain the ability to switch into a "hypoxic phenotype" as they transition to endothelial cells and adulthood.

Retinopathy of prematurity (ROP), a condition responsible for $13 \%$ of the cases of childhood blindness in the U.S. and $62 \%$ of the cases in Mexico, occurs because in utero blood oxygen levels are much lower than postnatal levels, disturbing vascular development among children born prematurely (Adams, 2008). The effect of this premature "relative hyperoxia" on angiogenesis is to downregulate hypoxia driven, VEGF mediated cell proliferation, resulting in delayed vascularization of the peripheral retina. Subsequent hypoxia in the peripheral retina then produces proliferation of blood vessels in the eye of the premature infant (Fleck and McIntosh, 2008). Children with ROP often display abnormal eye movements and crossed eyes, suggesting that developing periocular tissues are also sensitive to variations in oxygen tension (O'Connor et al., 2007). Also important to this study, a strong correlation has been found between strabismus, anisotropia, amblyopia, and microphthalmia among newborns and maternal smoking during pregnancy (Hakim RB, 1992; Lempert, 2005; Ponsonby AL, 2007; Stone RA, 2006). Smoking lowers maternal blood oxygen carrying capacity because carbon monoxide irreversibly binds to hemoglobin, thus there is a clear implication here that the fetus may be subjected to a relatively hypoxic environment when mom is a smoker.

Thus, hypoxia per se can not be said to precipitate abnormal development (Grabowski, 1958), but rather it provokes adaptive changes that occur in response to hypoxia, thereby changing the pattern of gene expression at critical periods (Seta and Millhorn, 2004). Embryonic stem cell populations do not all respond in the same way to hypoxia. The "hypoxic phenotype" 
among mesenchymal cells is characterized as "highly invasive and expressing several hypoxia regulated genes" (Lash et al., 2002). These features normally characterize trophoblast cells, that are responsive to hypoxic conditions via invasive, migratory behavior - if this behavior fails, abnormal blood flow occurs in the placenta's intervillous spaces as early as week 7 of gestation (Jaffe et al., 1997). Although relatively low oxygen tension is important for proper neurulation, autonomic nerves proliferate excessively along blood vessels among embryos experiencing chronic hypoxia (Ruijtenbeek et al., 2000), thus early peripheral nerve cell populations respond differently to hypoxia from neurepithial cells. Apoptosis and necrosis of brain tissue are among the most dramatic indicators of hypoxia among older embryos (Grabowski, 1966), demonstrating that nervous tissues' response to oxygen changes rapidly as they differentiate and grow. Among myoblasts, the two embryonic processes of cellular division and differentiation show reciprocal behaviors in response to oxygen. Although the rate of differentiation of myoblasts as measured by fusion into myotubes is proportional to oxygen concentration, the rate of division of myoblasts varies inversely with the oxygen concentration used, within a range of $2 \%-80 \%$ oxygen (Hollenberg et al., 1981).

The behavior of cells during cell migration and differentiation events is critical to proper tissue and organ assembly. A good example of a complex system consisting of different tissues that initially arise and proliferate in isolation from each other, but differentiate and grow in proximity, is the periocular region of the head. Vertebrate vision depends on the ability to stabilize the eye with respect to the surroundings long enough to generate an image on the retina. The oculorotatory muscles that perform this function commit to the myogenic lineage and are hard-wired to the brain very early, before migrating to their final periorbital positions, and while they are still in the paraxial mesoderm along the hindbrain of the embryo (Wahl, 2007). Eye muscles have been observed to develop even in the absence of eyes in some mutants, or where eye size is dramatically reduced (Franz and Besecke, 1991), an indication that the developmental program for early myogenic differentiation is not dependent on the presence of the eye. However, the periorbital environment is where extraocular muscles must integrate with surrounding support tissues and grow to appropriate size, so their ultimate functionality depends on the latter stages of organogenesis. The tissues that support the eyes and share that very limited periocular space include the optic nerve, lacrimal gland, extraocular muscles, fibroadipose tissue, peripheral nerves, ganglionic tissue, and blood vessels. These tissues originate both rostral, caudal, and dorsal to their final location in the periorbital region. They originate as neural crest, neural tube, ectodermal, and mesodermal cells.

\subsection{Effects of oxygen deprivation on craniofacial growth in chick embryos}

I designed experiments to study the physical environment's effects on early craniofacial development in chick embryos. My preliminary work is described here.

\subsubsection{Methods}

To learn how acute anoxia affects eye and periocular development, $48 \mathrm{hr}$ chick embryos (Hamburger-Hamilton stages 13-14) were exposed to a pure nitrogen atmosphere at the 
normal incubation temperature of $38^{\circ} \mathrm{C}$ for 2, 3, or 4 hours as follows: One cc of thin albumen was withdrawn from the pointed end of each egg using a sterile syringe. This eggshell opening was re-sealed with warm paraffin wax. A one-centimeter diameter window over the embryo was made by first cleaning the shell with $70 \%$ ethanol, allowing it to dry, and then chipping away the shell using sterile forceps. Embryos were examined and staged according to the Hamburger-Hamilton stage series (HH). Any embryos found to be developing abnormally were eliminated from the experiment, the $\mathrm{HH}$ stage of each remaining normal embryo was recorded in pencil on each egg, and the eggshell window was sealed with clear Scotchgard tape. Eggs were transferred to Billups-Rothenburg incubator pods. One pod was flushed for two minutes with high-purity nitrogen gas, and sealed for either 2, 3, or 4 hours. Normal atmospheric air was left in the other (control) pod. Eggs were then returned to a standard, humidified Percival incubator with circulating atmospheric air and allowed to continue developing normally for an additional 2 days. Embryos were examined in situ, then collected into $4 \%$ paraformaldehyde in phosphate buffer ( $\mathrm{pH}$ 7.4) for further study.

\subsubsection{Results}

I found that hypoxia causes craniofacial malformations of increasing severity, proportional to the length of exposure to anoxic conditions (pure nitrogen gas). Most (95\%) of both control and experimental embryos survived and were robustly vascularized. A composite photo of representative embryos, placed over a micrometer ruler, is shown in Figure 2. Compared to control embryos (A), 3-hour exposure to anoxic conditions produced ocular phenotypes varying from near-normal to microphthalmic (B), and more than half of all embryos in this group were reduced in size compared to the controls. Four hours of anoxia produced $100 \%$ anencephalic, dwarfed embryos (C). Two hours of anoxia resulted in grossly normal embryos (data not shown).

Frontal development of the face in each of these treatments is shown in Figure 3. Normallydeveloping embryos (A) display prominent medial nasal and maxillary prominences, and the lateral nasal prominence is also well-developed. After 3 hours of anoxia, the maxillary process is reduced or absent (B) and the eyes are smaller than normal. These deformities are not bilaterally symmetrical in every case, as shown in B. The ocular defect includes a lens that is disproportionately large relative to the eyecup. After 4 hours of anoxia, all embryos exhibit anencephalia, but some retain tissues from the lower face (C). At the time of treatment, at stage 14, the primary eye field has already separated, embryos have developed eyecups, and their lens placodes are in the process of invaginating to form vesicles. In $\mathrm{C}$, it can be seen that after 4 hours of anoxia, eyecups subsequently failed to expand. However, lens vesicles did form (arrow). The eyecups differentiated further, but failed to grow: pigmented epithelium extends along the presumptive optic tract. This abnormal distribution of pigmented cells indicates defects in genetic patterning that should have separated the eyecup from the optic tract and brain.

All surviving embryos had well-developed vitelline vasculature, and normal trunk and limb morphology. Several of those exposed to nitrogen had avascular allantoic membranes. 


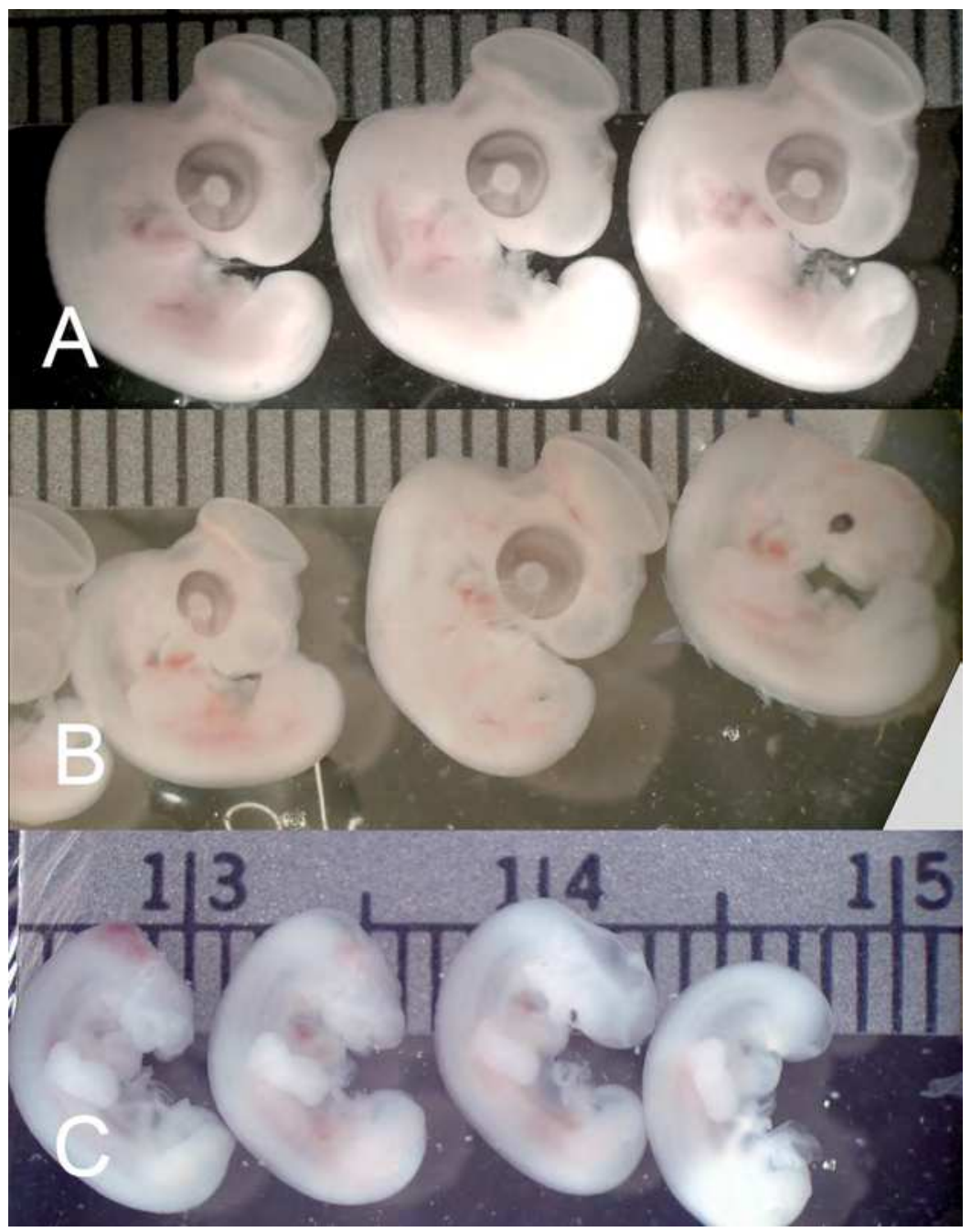

Fig. 2. Lateral view of chick embryos exposed at $\mathrm{HH}$ stage 14 (48 $\mathrm{hr}$ incubation) to nitrogen gas for 0 (A), 3 hours (B), or 4 hours (C), then returned to normal atmospheric conditions and allowed to develop for a further 2 days. To provide scale, the embryos are positioned over a centimeter ruler. 


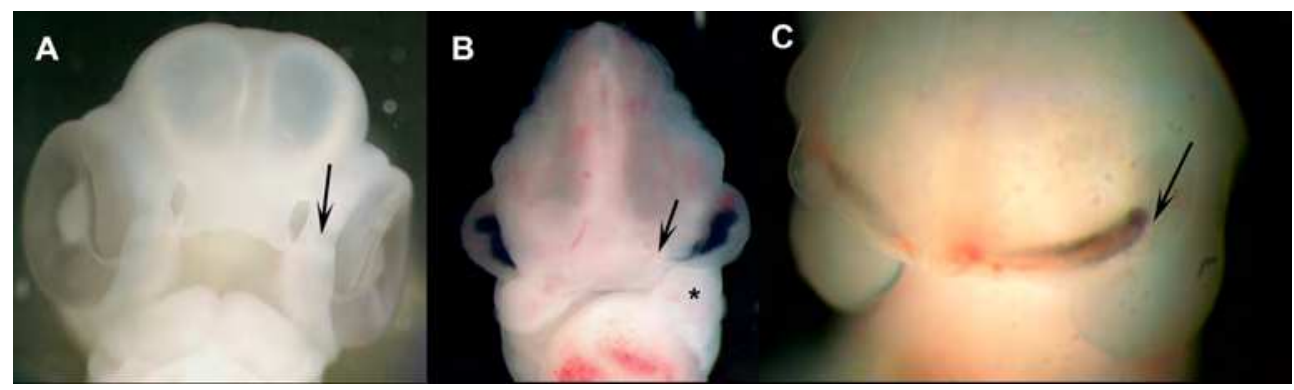

Fig. 3. Frontal views at 4 days: (A) control embryos (HH stage 25). Medial and lateral nasal processes are fusing with the maxillary process (arrow). Eyes have expanded greatly since stage 14, when the lens placode was forming a vesicle and inducing formation of the optic cup. (B) Failure of the left maxillary process to develop after 3 hours of anoxia at stage 14. Only the medial and lateral nasal processes are intact (arrow), and the nasal pits are reduced in size. An undivided visceral arch is present (asterisk). Partial fusion of the undivided first arch is seen, with the medial/lateral nasal processes on the right. Reduction in size of the frontonasal prominence of the neural tube, and failure of the eyecups to expand is apparent. Lens vesicles have formed. (C) Following 4 hours of anoxia, the frontonasal prominence is entirely absent. The first arch has not divided (it is located just below the arrow).

The eyecups have failed to expand. Differentiation has proceeded, but genetic programming that should distinguish and separate the eyes from the optic tract has failed, as demonstrated by a trail of pigmented epithelium that extends along the entire presumptive optic tract. Lens vesicles have formed (arrow).

\subsubsection{Conclusions}

Growth and differentiation of the eyecup, brain, and first visceral arch is retarded if exposed to anoxic conditions at $\mathrm{HH}$ stages 13 or 14 . The first arch fails to properly divide and grow, resulting in severe reduction of the maxillary process on one or both sides. However, the lens placode does form a lens vesicle and the olfactory placode develops into a nasal pit. Histological assessment is necessary to determine whether this stunted growth is a result of necrosis or arrested mitosis, and to follow the differentiation of the periocular mesenchyme and muscles. Morphometry of the defect at different exposures at different developmental intervals will provide a trajectory of severity that can be analyzed to determine the relative susceptibility of each cell population contributing to the growth of the face.

\subsection{Effects of light on growth and shape of the eye}

It is a common misperception that the lens and cornea display fixed patterns of development that are independent of non-visual environmental influence, however we have found that light regimen plays an important role in overall shaping of the eye. The effect of light on the cornea, in particular, is of interest because the air/cornea interface is the major focusing surface of the eye. The development of persistent ocular defocus is commonly studied in the chick (Gottlieb et al., 1987; Wallman et al., 1978). Refractive errors (myopia, or nearsightedness, hyperopia, or farsightedness) have been induced in chick eyes using constant darkness (CD, (Gottlieb et al., 1987), and constant light (CL, (Lauber et al., 1970); 
(Li, 1995)), producing corneal flattening and hyperopia within three weeks. Long term CL produces shallow anterior chambers, corneal thickening, lenticular thinning, cataracts, and damage to the retina, pigment epithelium, and choroid (Li, 1995).

These studies demonstrate that corneal shaping during growth is influenced by ambient light. We found differences in the pattern of corneal growth between chicks raised in CL vs. normal light conditions ( $\mathrm{N}$, raised in 12 hours light/12 hours darkness) using morphometric techniques, including: a) a comparison of eye weights and wet and dry corneal weights, b) measurement of corneal thicknesses and corneal diameters, c) spatial dynamics of corneal expansion, d) measurement of corneal curvatures, and e) stromal cell densities (Wahl, 2009). We learned that the eye's ability to model its shape towards emmetropia is diminished in the absence of periods of light and dark. Particularly sensitive are the stromal cells of the cornea, which show significant changes in density and distribution in CL.

We pursued this finding with additional experiments to learn whether the effect of CL was a direct result of light on the corneal cells, or whether stromal growth of the cornea was regulated by hormones that, in turn, were affected by light cycle (Wahl, 2011). To do this, an organ culture system was designed for chick corneas. Light regimen alone had no effect on corneal growth in culture. Melatonin and/or retinoic acid were applied to the cornea both in vivo or in vitro, and compared to controls. We found that both melatonin and retinoic acid affect the hydration state of the cornea and alter its shape in growing birds, and we speculate that this effect results from altered ratios of glycosaminoglycans (GAGs) in the corneal matrix. It has been demonstrated that the corneal matrix has a gradient of GAGs that have different properties of hydration (Castoro, 1988), and so it is reasonable to suppose that altering this gradient or changing the ratio of GAG production in any way could affect the curvature of the cornea and its thickness.

\section{Morphometric changes in response to physical/mechanical injury}

Embryos have a remarkable ability to regenerate themselves through re-specification of cell populations, often resulting in a change in shape and/or body mass. They do not scar, however at birth they are usually smaller and may be physically disproportionate.

\subsection{Chick embryo regenerative capacity}

Surgical manipulation of avian embryonic tissues always introduces a greater number of variables than the experimenter can control for or, often, readily identify. Because most of our microscopic approaches to the study of embryonic cell behavior, individually or collectively, is limited by the necessity of killing the cells, we really have very little concept about how these cells are dynamically interacting, or what timeframe is involved in those interactions. Most analyses of avian embryonic development are devoted to defining normal events, especially identifying the origins of specific tissues and documenting the precise history and movements of cellular precursors. The observational skills required for this work include morphometric tools that allow interpretation of relationships among tissues surrounding the site or sites of interest. It is important to be prepared for unexpected findings in these studies, as it is all too easy to shoehorn one's observations to fit into a 
popular theory, rather than consider the possibility that something entirely new is being witnessed.

In the quest to follow the fates of individual precursor cells in chick embryos, the most significant technical advancement was the discovery by Nicole LeDouarin of a nucleolar marker present in most quail cells (Douarin and Barq, 1969). Staining for nucleolarassociated heterochromatin in quail cells allows transplanted quail cells and all their progeny to be followed in avian embryos throughout their development. The quail-chick chimeric method has been applied to nearly all developing organ systems (Wahl and Noden, 2001) but it is in following the fates of highly migratory populations such as the neural crest, myoblasts, angioblasts, and gastrulating mesoblasts that the greatest benefits have accrued.

Morphometric assessment may be made using these methods. Questions such as what number of cellular progeny are produced, how far and in what directions they have moved, and what three-dimensional changes in shape follow a specific time interval or manipulation may be addressed using specific lineage tracing techniques.

One typical method in embryology involves ablation of a target tissue of interest. In many situations, ablations are repaired by compensatory hyperplasia and restitution of the deleted tissue by remaining committed progenitors or adjacent multipotent cells. Healing without restitution, as in the case of an ablated optic cup, may indicate an absence of nearby responsive multipotent populations, or inhibition by newly-differentiated neighboring cells, such as occurs between rhombomeres (Guthrie and Lumsden, 1991). Where restitution takes place, the regenerated element is generally smaller than the normal counterpart, an effect that becomes increasingly pronounced as the age of the embryo at the time of ablation increases.

During transplant procedures, both the size and shapes of the graft and the host lesion sites often change considerably within minutes of excising the tissue. Surface tension at the wound margin contributes to this, both expansive (e.g. surface ectoderm) and compressive (e.g. neural plate). Usually, these changes are transient, however during the initial healing-in time they can be quite important. Many of us have spent hours struggling to fit a curling graft precisely into a well-cut host hindbrain, as the margins of the host site begin to shrink and the graft, too, becomes more compact. If the embryo appears healthy several hours after tissue transplantation and grafted tissue is evident at the intended location, then the surgery is considered a success. If the embryo is alive and shows no gross abnormalities after several days, all the better! However, this 'normalcy' may mask substantial transient or permanent deviations from the normal course of development.

In our experiments on several embryonic tissues in the neurula-stage avian head, we assume that all cells that are directly contacted by microsurgical instruments die immediately. Even among embryos that appear to heal excellently, extensive cellular disintegration adjacent to the lesion is evident via histological examination within a few hours of surgery. This focal cellular trauma can initiate responses that alter the normal intra-embryonic milieu at considerable distances from the site of surgery.

We also found that focal cellular trauma can initiate responses that alter the normal behavior of cells at some distance from the surgical site. In particular, nerve trajectories were 
disturbed as far away as the forebrain following a lesion in the hindbrain (Wahl and Noden, 2001). Careful morphometry and assessment at multiple times following surgery are important to proper understanding of this phenomenon.

\subsection{Salamander regenerative ability following gross physical injury}

Most urodeles can regenerate many body tissues, including most of the eye. Structures that regenerate include the retina, the lens, the iris, the pigment epithelium (RPE), and the choroid. Tissue replacement may even be repetitive (Hasegawa, 1965; Reyer, 1977a; Stone, 1960), but the mechanism involved is incompletely understood. Most investigators agree that the central retina is regenerated from the RPE, while the periphery is replaced by cells of the pars-ciliaris-ora serrata complex (Hendrickson, 1964; Keefe, 1973).

During regeneration several processes occur simultaneously, e.g. necrosis triggers phagocytosis by migrating macrophages, the eye's dimensions diminish as the vitreous cavity shrinks, and normally, the lens deteriorates. There is a concurrent proliferation of cell types that ultimately restores function to the eye: cells destined to from new lens, new RPE, and new retina appear and may migrate to sites of continued development. Even cell death among regenerating cells may further affect the changing morphology of the eye (Oppenheim, 1981).

Neural retina regeneration in larval Triturus. pyrrogaster and T. viridescens, as in adult newts, was initiated primarily at the growth zone of the anterior complex. Larval urodelan eyes, unlike the eyes of adults, are resistant to a temporary loss of blood supply and can be transplanted without a degeneration of the neural retina (Stone, 1930). Regeneration is more rapid in larvae than adults, and is initiated exclusively from the peripheral margins of the retina. In larval Ambystoma maculatum lentectomy and retinectomy result in regeneration from the marginal growth zone as in T. viridescens (Stone and Cole, 1942; Stone and Ellison, 1945). However, neural retina regeneration did not occur over a waiting period of sixty days when only the retinal pigment epithelium was left in the eye.

Comparing regenerative events of newt limbs with those of the eye is relevant (Zarrow, 1961). During the first stage of limb regeneration, the wound is covered by a specific wound epithelium without which regrowth will not occur. This special epithelium is known as the "wound blastema". A sutured wound will not regenerate...it requires the wound blastema to organize regrowth of the missing tissue(s). Initially, this epithelium is translucent, and later becomes pigmented. It is formed by a single layer of cells that migrate from the periphery of the wound. This regenerative layer later proliferates, becoming up to several cell layers thick, and displays extensive mitotic activity. Initially, the epidermal cells are squamous, becoming columnar as they proliferate. Later they are almost exclusively cuboidal. The basal layers, however, remain low columnar (Zarrow, 1961). The basal cells of the wound epithelium form villous projections into the subjacent dermis. In normal dermis, the reticular basement membrane forms a coarse network through which migratory (macrophage) cells move with relative ease, extending their pseudopodia between the fibers. Epithelization of the wound is followed by a random deposition of fibrils basally that in form resembles a feltlike mat, similar to the normal dermis. Later, lamellar organization and differentiation occur. 


\section{Morphometry may be the principle way to solve certain developmental problems: 3-D analysis of primordial follicle distribution}

Some problems in development are best solved using morphometric analysis. A prime example of this is the ongoing question of ovarian follicular reserves. A "central dogma" of female reproductive biology has long held that oogenesis ceases prior to birth in most mammals and that the functional lifespan of the ovaries is dictated in part by the number of oocytes present; a number that is known to decline precipitously during both fetal development and postnatal life. Primordial (dormant) follicles are distributed in an apparently random fashion throughout the ovarian outer cortex during the three-day estrus cycle of the mouse. However, a discrepancy of 10-fold or more has been shown in the total numbers of follicles among individuals and among mouse strains (Bolon et al., 1997; Bucci et al., 1997). Most consider this a failure of the sampling methods, and call for a more reliable way to evaluate ovarian follicular reserves using a standardized procedure. Most reported methods employ sampling of the ovary by counting representative sections. They use this data to calculate the number of follicles per representative volume, and then multiply that figure by the total volume of the ovary under study(Britt et al., 2004). However, it is difficult to see how one can improve on total sampling of the ovarian reserve, since there are widely different follicle populations among different strains and ages of mice (Myers et al., 2004). This fact, in addition to evidence that replacement germ cells may exist in the bone marrow (Tilly, 2003), suggest that gametes may arise from a more complicated stem cell population than long supposed.

An alternative hypothesis for such variation could be that the population of follicles in the mouse ovary is dynamic, and is in fact replenished by as yet undetermined mechanisms.

We designed a three-dimensional reconstruction method to accurately portray primordial follicle distributions in young mouse ovaries. We reasoned that primordial follicles are not randomly distributed throughout the ovarian cortex, and wished to visualize the variation in follicle distribution in the cortex from ovary to ovary. Our previously unpublished work is presented here.

\subsection{Specimen preparation and histological assessment}

Three "wild-type mice" were raised until 5 months of age and euthanized by $\mathrm{CO}_{2}$ overdose during the same stage of estrus. Their left ovaries were fixed in Bouin's fixative and then paraffin embedded. The tissues were serially sectioned at $6 \mu \mathrm{m}$ and stained with Periodic Acid Schiff (PAS) and iron hematoxylin.

Histological examination of the stained and sectioned ovaries showed good preservation of tissue structure and normal ovarian anatomy. However, rare clusters of primordial oocytes sharing a single follicle were found (Figure 4). I have found no reference to the occurrence of follicles with multiple oocytes in the literature. I suspect we found these rare follicles because we were very thorough in our examination of every section from each ovary. We found just two such follicles in the three ovaries reconstructed in Figure 5, and no more than three among several other ovaries not included in this study. These unusual compound follicles are very interesting, although their rarity is an obstacle to further study. 


\subsection{Analysis of primordial follicular distribution}

Using a Zeiss microscope equipped with a camera lucida, tracings of every section within each ovary were made at 100X magnification. Each tracing delineated the boundary of the ovary as well as the location of primordial follicles within that section. Since each follicle occupied more than one section, primordial follicles were defined in this study as those sections containing the nucleus of the oocyte, and surrounded by a single layer of predominantly squamous granulosa cells, of which no more than fifty percent were cuboidal. We used the tracings to map the coordinates of each primordial follicle in threedimensional space using SYSTAT with the ovary slice number as the Z coordinate.

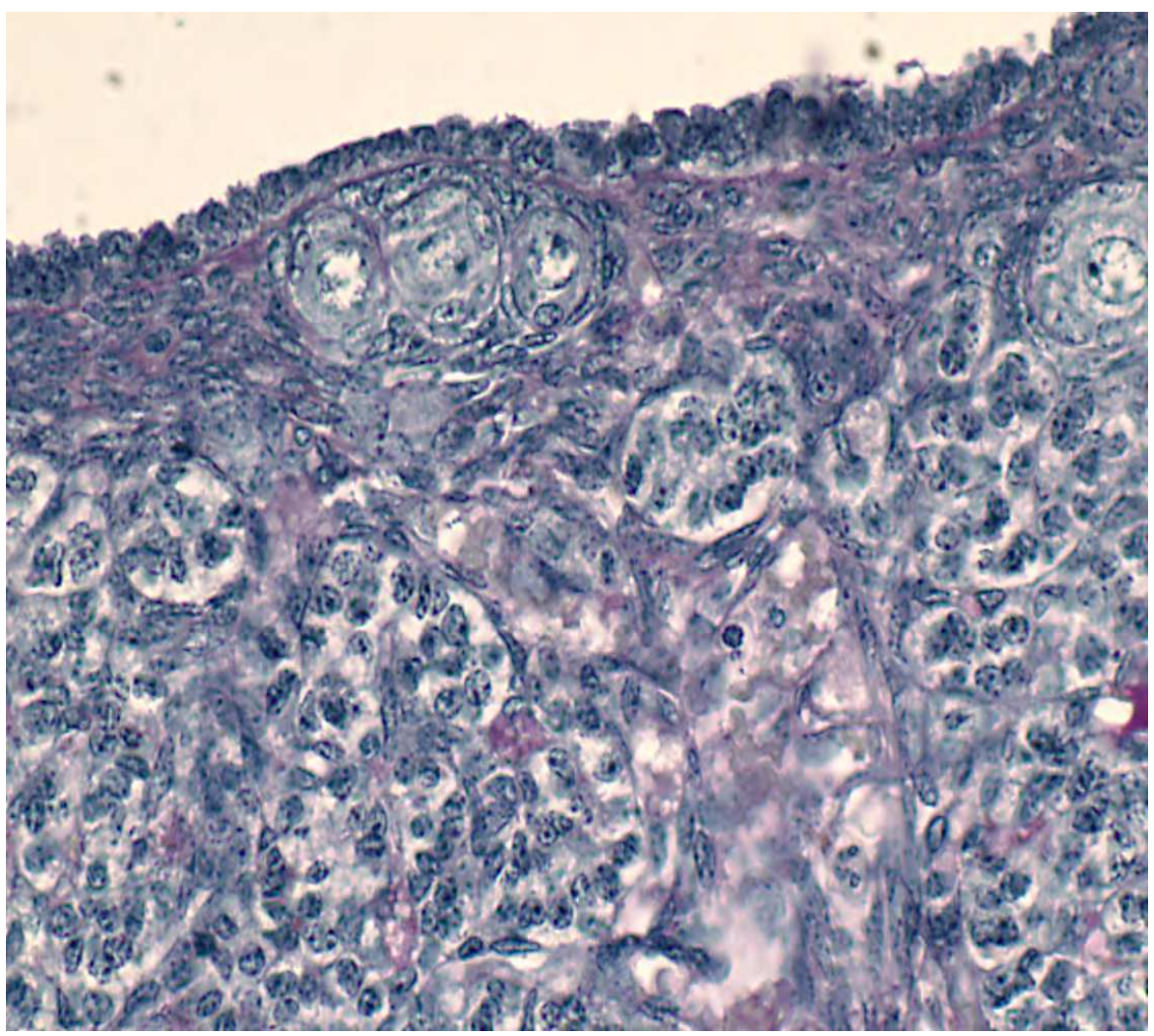

Fig. 4. Cluster of three oocytes within a single primordial follicle from a 6 micron paraffin section stained with iron hematoxylin and PAS. Primordial oocyte clusters are not discussed in the literature, however we see them occasionally. They are usually located near the germinal epithelium, as seen here. 


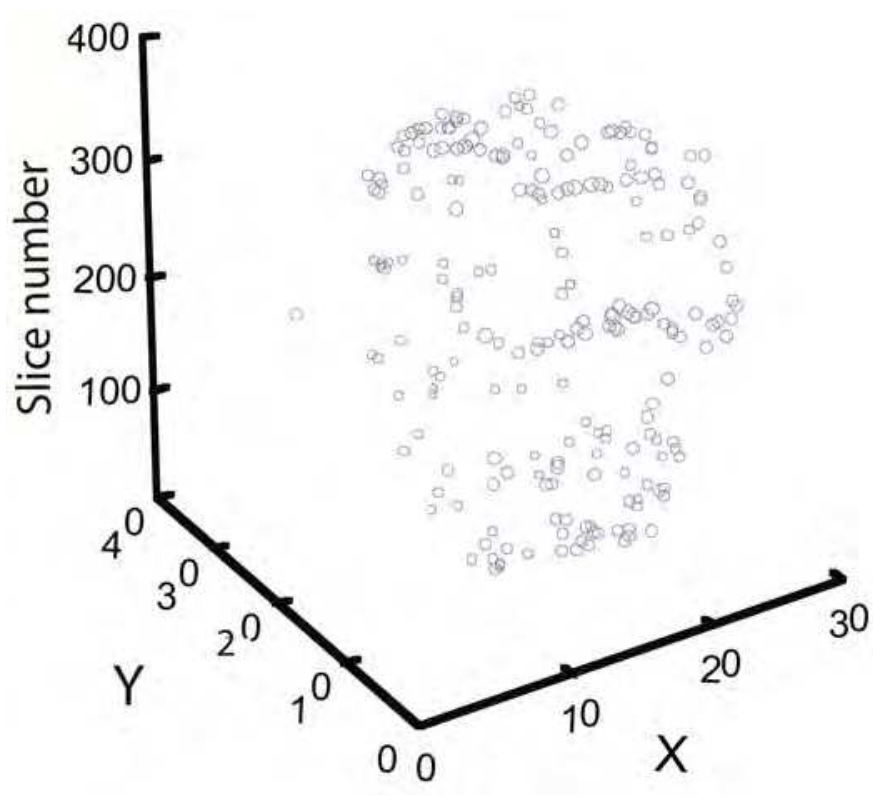

A

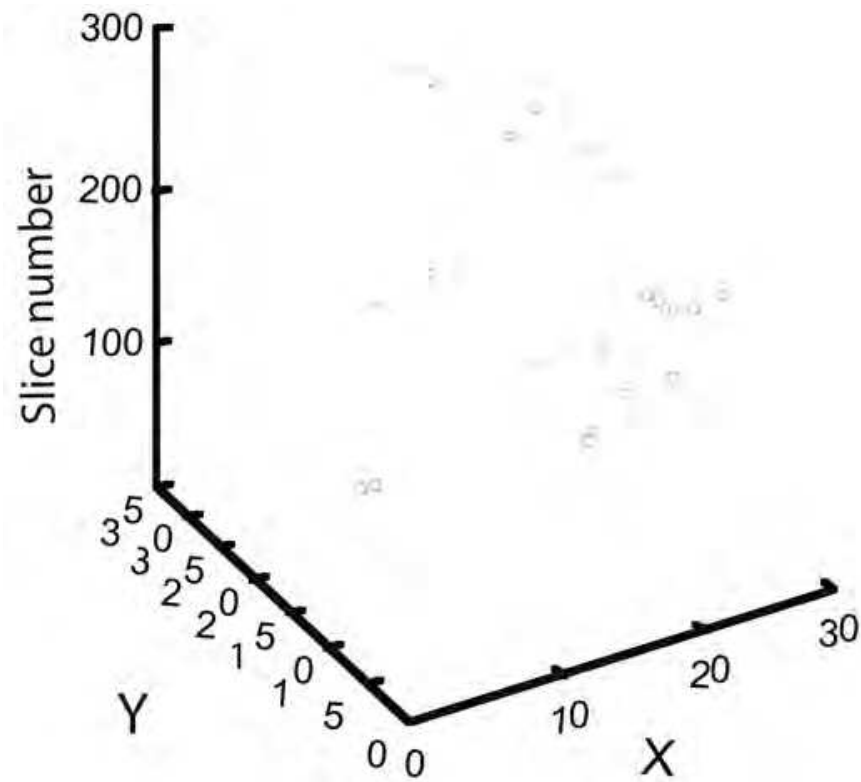

B 


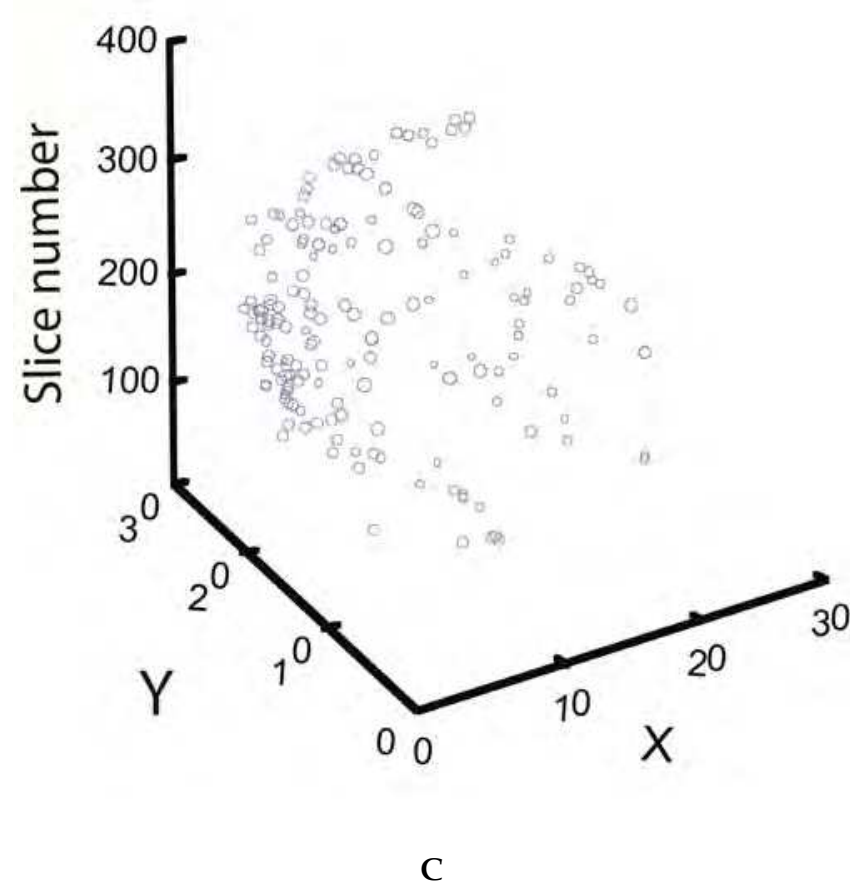

Fig. 5. 3-D reconstruction of primordial follicle distribution within three mouse ovaries. Ovary A. Coefficient of Dispersion $=2.30$, Ovary B. Coefficient of Dispersion $=1.28$, Ovary C. Coefficient of Dispersion $=4.05$. In this representation, follicles that are near the observer in the $\mathrm{Z}$ axis are shown as large circles, whereas those further away are small circles. Note that ovary B has far fewer follicles than either A or C, although all three were from 5 month old female mice that came from the same litter. Note that none of these have random distributions of follicles, but rather, the follicles occur in clumped patterns.

Each ovary drawing was divided into approximate cubic units $(350 \times 350 \times 300 \mu \mathrm{m})$, and then the number of primordial follicles in each cube was recorded, discounting cubes containing the medulla or corpora luteae. We graphed the positions of primordial follicles among cubes using a modified Poisson distribution, and then calculated a coefficient of dispersion by finding the ratio of the variance of numbers of follicles per cube to the mean number per cube.

A coefficient of dispersion greater than 1 is indicative of a clumped distribution pattern. All three ovaries had a coefficient of dispersion greater than 1, thus we conclude that primordial follicles are non-randomly distributed in the ovarian cortex. Also apparent 
from the figures is that the number of primordial follicles varies widely from one ovary to the next.

From just these three reconstructions, it may be seen that a) there are not enough primordial follicles in the 5 month mouse ovary to account for the number required throughout its reproductive lifespan, b) primordial follicles are not randomly dispersed throughout the ovary, and c) numbers of follicles vary widely from one mouse to the next, although all three were collected while in the same phase of the estrus cycle. These observations demonstrate the power of careful morphometry in elucidating important, fundamental facts about the basic biology of the organism that are difficult to obtain any other way.

\section{Summary}

In this paper, I have tried to provide a sense of the great range of morphological plasticity in developing systems... both plasticity of normal development, and in response to injury or environmental change. In addition to a brief review of the literature, I have used examples from my own work (both published and unpublished) to illustrate the plasticity of form among a wide variety of vertebrate embryos, and I have indicated how morphometric analysis is a useful tool for learning about the changes of form possible in developing vertebrates. The emergent properties of the developing organism, both in response to the environment, or following injury, illustrate yet again that in biological systems, the whole is always greater than the sum of its parts.

\section{Acknowledgments}

I wish to thank the following individuals who participated in different parts of the original research projects reported here: Howard C. Howland, Dept. of Neurobiology and Behavior, Cornell University, Allison Inga, former Wells College undergraduate and now student of veterinary medicine at Ross University, Drew M. Noden, Department of Biomedical Sciences, Cornell University, and Yuko Takagi, former Wells College undergraduate and now postdoctoral associate at Harvard University School of Medicine. I also thank Wells College for providing space and opportunities for undergraduates to pursue original research, and the NIH for funding that supported my work on eye growth. Finally, I wholeheartedly thank my husband, Ellis Loew, Department of Biomedical Sciences, Cornell University, for help in reviewing this chapter, and for tolerating household mayhem while I wrote it.

\section{References}

Adams G (2008) Editorial on retinopathy of prematurity. Early Human Development 84:75-76

Bolon B, Bucci TJ, Warbritton AR, Chen JJ, Mattison DR, Heindel JJ (1997) Differential follicle counts as a screen for chemically induced ovarian toxicity in mice: results from continuous breeding bioassays. Fundamentals of Applied Toxicology 39:1-10 
Bonner JT (1969) On Growth and Form, by D'Arcy Wentworth Thompson. Cambridge University Press

Boughner JD, Wat S, Diewert VM, Young NM, Browder LW, Hallgrímsson BJ (2008) Shortfaced mice and developmental interactions between the brain and the face. Journal of Anatomy 213:646-662

Britt KL, Ebling FJP, Kerr JB, Myers M, Wreford NGM (2004) Methods for quantifying follicular numbers within the mouse ovary. Reproduction 127:569-580

Bucci TJ, Bolon B, Warbritton AR, Chen JJ, Heindel JJ (1997) Influence of sampling on the reproducibility of ovarian follicle counts in mouse toxicity studies. Reproductive Toxicology 11:689-696

Buresova M, Zidek V, Musilova A, Simakova M, Fucikova A, Bila V, Kren V, Kazdova L, Di Nicolantonio R, Pravenec M (2006) Genetic relationship between placental and fetal weights and markers of the metabolic syndrome in rat recombinant inbred strains Physiol Genomics 26:226-231

Castoro JA, A. A. Bettelheim, et al (1988) Water gradients across bovine cornea. Investigative Opthalmology and Visual Science 29:963

Douarin NML, Barq G (1969) Use of Japanese quail cells as 'biological markers' in experimental embryology. C R Acad Sci Hebd Seances Acad Sci D 269:15431546

Etcoff N (2000) Survival of the Prettiest: The Science of Beauty. Abacus Books, London

Fleck B, McIntosh N (2008) Pathogenesis of retinopathy of prematurity and possible preventive strategies. Early Human Development 84:83-88

Franz T, Besecke A (1991) The development of the eye in homozygotes of the mouse mutant extra- toes. Anat EMBRYOL 184:355-362

Gottlieb M, Wentzek L, Wallman J (1987) Different visual restrictions produce different ametropia and different eye shapes. Investigative Ophthalmology and Visual Science 28:1225-1235

Gould SJ (1966) Allometry and size in ontogeny and phylogeny. Biological Reviews 41:587-640

Grabowski CT (1966) The etiology of hypoxia-induced malformations in the chick embryo. Journal of Experimental Zoology 157:307-326

Grabowski CT, Paar, John A. (1958) The teratogenic effects of graded doses of hypoxia on the chick embryo. The American Journal of Anatomy 103:313-347

Guthrie S, Lumsden A (1991) Formation and regeneration of rhombomere boundaries in the developing chick hindbrain. Development 112:221-229

Hakim RB TJ (1992) Maternal cigarette smoking during pregnancy. A risk factor for childhood strabismus. Arch Ophthalmol 110:1459-1462

Hall BK, Herring SW (1990) Paralysis and growth of the musculoskeletal system in the embryonic chick. Journal of Morphology 206:45-56

Hasegawa M (1965) Restitution of the eye from the iris after removal of the retina and lens together with the eye-coats in the newt, Triturus pyrrhogaster. Embryologia 8:362-386

Hendrickson A (1964) Regeneration of the retina in the newt Diemictylus v. viridescens. University of Washington 
Hollenberg M, Honbo N, Ghani QP, Samorodin AJ (1981) Oxygen enhances fusion of cultured chick embryo myoblasts. . Journal of Cellular Physiology 106:10974652

Huang S-TJ, Vo KCT, Lyell DJ, Faesen GH, Tulac S, Tibshirani R, Giaccia AJ, Giudice LC (2004) Developmental response to hypoxia. The FASEB Journal 18:1348-1365

Jaffe R, Dorgan A, Abramowicz JS (1997) Maternal circulation in the first-trimester human placenta--myth or reality? American Journal of Obstetrics and Gynecology 176:695-705

Keefe JR (1973) An analysis of urodelian retinal regeneration: IV. Studies of the cellular source of retinal regeneration in Triturus cristatus carnifex using H3-thymidine. Journal of Experimental Zoology 184:239-258

Klingenberg CP (2003) A developmental perspective on developmental instability: theory, models, and mechanisms. In: Polak M (ed) Developmental Instability: Causes and consequences Oxford Press, New York

Klingenberg CP (2008) Morphological integration and developmental modularity. Annu Rev Ecol Evol Syst 39:115-132

Lash GE, Postovit L-M, Matthews NE, Chung EY, Canning MT, Pross H, Adams MA, Graham $\mathrm{CH}$ (2002) Oxygen as a regulator of cellular phenotypes in pregnancy and cancer. Canadian Journal of Physiology and Pharmacology 80:103-109

Lauber J, Boyd J, Boyd T (1970) Intraocular pressure and aqueous outflow facility in lightinduced avian buphthalmos. Experimental Eye Research 9:181-187

Lempert P (2005) Amblyopia pervalence and cigarette smoking by women. Opthalmic Physiol Opt 25:592-595

Li T, D. Troilo, et al (1995) Constant light produces severe corneal flattening and hyperopia in chickens. Vision Research 35(9):1203-1209

McCune AR (1981) Quantitative description of body form in fishes: Implications for species level taxonomy and ecological inference. Copeia 1981:897-901

Mitashov VI (1978) Replacement of melanin granules in the iris and pigment epithelium of the retina in adult newt after completion of eye regeneration. Soviet Journal of Developmental Biology 9:150-155

Müller GB (2003) Embryonic motility: environmental influences and evolutionary innovation. Evolution and Development 5:56-60

Müller GB (2011) BIO. Evolution and Development 13:243-246

Myers M, Britt KL, Wreford NGM, Ebling FJP, Kerr JB (2004) Methods for quantifying follicular numbers within the mouse ovary. Reproduction 127:569-580

O'Connor AR, Wilson CM, Fielder AR (2007) Opthalmological problems associated with preterm birth. Eye 21:1254-1260

Oppenheim RW (1981) Neuronal cell death and some related regressive phenomena during neurogenesis: A selective historical review and progress report. In: Cowan WM (ed) Studies in developmental neurobiology; essays in honor of Viktor Hamburger. Oxford Press, N.Y., pp 74-133

Ponsonby AL BS, Kearns LS, MacKinnon JR, Scotter LW, Cochrane JA, Mackey DA (2007) The association between maternal smoking in pregnancy, other early life 
characteristics and childhood vision: the Twins Eye Study in Tasmania. Ophthalmic Epidemiol 14:351-359

Provot S, Zinyk D, Gunes Y, Kathri R, Le Q, Kronenberg HM, Johnson RS, Longaker MT, Giaccia AJ, Schipani E (2007) Hif-1a regulates differentiation of limb bud mesenchyme and joint development. Journal of Cell Biology 177:451-464

Reyer R (1977a) Repolarization of reversed, regenerating lenses in adult newts Notophthalmus viridescens. Experimental Eye Research 24:501-509

Reyer RW (1977b) The amphibian eye: Development and regeneration. Handbook of Sensory Physiology, pp 309-390

Ruijtenbeek K, le Noble FAC, Janssen GMJ, Kessels CGA, Fazzi GE, C.E. B, De Mey JGR (2000) Chronic hypoxia stimulates periarterial sympathetic nerve development in chicken embryo. Circulation Research 102

Serrat MA, King D, Lovejoy CO (2008) Temperature regulates limb length in homeotherms by directly modulating cartilage growth. PNAS 105:19348-19353

Seta KA, Millhorn DE (2004) Functional genomics approach to hypoxia signaling. Journal of Applied Physiology 96:765-773

Stone LS (1930) Heteroplastic transplantation of eyes between the larvae of two species of Amblystoma. J Exp Zool 55:193-261

Stone LS (1960) Regeneration in the lens, iris, and neural retina in a vertebrate eye. Yale Journal of Biology and Medicine 32:464-473

Stone LS, Cole CH (1942) Grafted eyes of young and old adult salamanders (Amblystoma punctatum) showing return of vision. Yale Journal of Biolgy and Medicine 15:735-754

Stone LS, Ellison FS (1945) Return of vision in eyes exchanged between adult salamanders of different species. Journal of Experimental Zoology 100:217-227

Stone RA WL, Ying GS, Liu C, Criss JS, Orlow J, Lindstrom JM, Quinn GE (2006) Associations between childhood refraction and parental smoking. Invest Ophthalmol Vis Sci 47:4277-4287

Thompson TJ, Owens PDA, Wilson DJ (1989) Intramembranous osteogenesis and angiogenesis in the chick embryo. Journal of Anatomy 166:55-65

Tilly JL (2003) Ovarian follicle counts--not as simple as 1,2,3. Reproductive Biology and Endocrinology 1:11

Van Valen L (1962) A study of fluctuating asymmetry. Evolution 16:125-142

Wahl C (2007) Periocular Mesenchyme: The interactions of neural crest and mesoderm. In: Tasman W, Jaeger EA (eds) Duane's Foundations of Clinical Ophthalmology. Harper and Row

Wahl CM (1985) SEM study of photoreceptor differentiation in regenerating retinas of the newt N. viridescens. Physiology. Cornell, Ithaca, NY

Wahl CM, Li, T.,Choden,T., Howland, H.C. (2009) Morphometrics of corneal growth of chicks raised in constant light. Journal of Anatomy 214:355-361

Wahl CM, Noden DM (2001) Cryptic responses to tissue manipulations in avian embryos. International Journal of Developmental Neuroscience 19:183-196

Wahl CM, T. Li, et al (2011) Effects of light and melatonin on chick corneas grown in culture. Journal of Anatomy in press 
Wallman J, Turkel J, Trachtman J (1978) Extreme Myopia Produced by Modest Change in Early Visual Experience. Science 201:1249-1251

Webster KA (2007) Hypoxia: Life on the Edge. Antioxidants \& Redox Signaling 9:1303-1307

Wells JC (2007) The thrifty phenotype as an adaptive maternal effect. Biol Rev Camb Philos Soc 82:143-172

Yamada T, Dumont JN, Moret, Brun (1978) Autophagy in dedifferentiating newt iris epithelial cells in vitro. Differentiation 11:113-147

Zarrow MXe (1961) Growth in Living Systems. Basic Books, New York. 


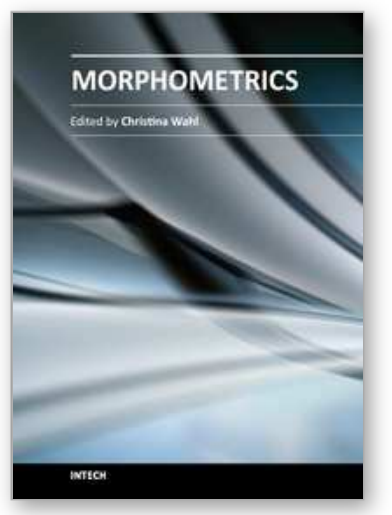

\author{
Morphometrics \\ Edited by Prof. Christina Wahl
}

ISBN 978-953-51-0172-7

Hard cover, 108 pages

Publisher InTech

Published online 02, March, 2012

Published in print edition March, 2012

It is human nature to measure things, and this holds true for science as well as everyday life. The five papers in this book demonstrate the usefulness of a morphometric approach to a variety of subjects in natural history, including systematics, phenotypic plasticity in response to environmental variation, and ontogenetic adaptation. As our understanding of genetic control mechanisms and epigenetics has matured over the last several decades, it has become clear that morphometric assessment continues to be important to our overall understanding of natural variability in growth and form. The tremendous growth of our knowledge base during the last century has necessitated that we find new ways to measure and track greater detail as well as greater numbers of parameters among populations and individuals.

\title{
How to reference
}

In order to correctly reference this scholarly work, feel free to copy and paste the following:

Christina Wahl (2012). Morphometry Applied to the Study of Morphological Plasticity During Vertebrate Development, Morphometrics, Prof. Christina Wahl (Ed.), ISBN: 978-953-51-0172-7, InTech, Available from: http://www.intechopen.com/books/morphometrics/morphological-plasticity-during-development

\section{INTECH}

open science | open minds

\section{InTech Europe}

University Campus STeP Ri Slavka Krautzeka 83/A 51000 Rijeka, Croatia Phone: +385 (51) 770447 Fax: +385 (51) 686166 www.intechopen.com

\section{InTech China}

Unit 405, Office Block, Hotel Equatorial Shanghai No.65, Yan An Road (West), Shanghai, 200040, China 中国上海市延安西路65号上海国际贵都大饭店办公楼405单元 Phone: +86-21-62489820

Fax: $+86-21-62489821$ 
(C) 2012 The Author(s). Licensee IntechOpen. This is an open access article distributed under the terms of the Creative Commons Attribution 3.0 License, which permits unrestricted use, distribution, and reproduction in any medium, provided the original work is properly cited. 